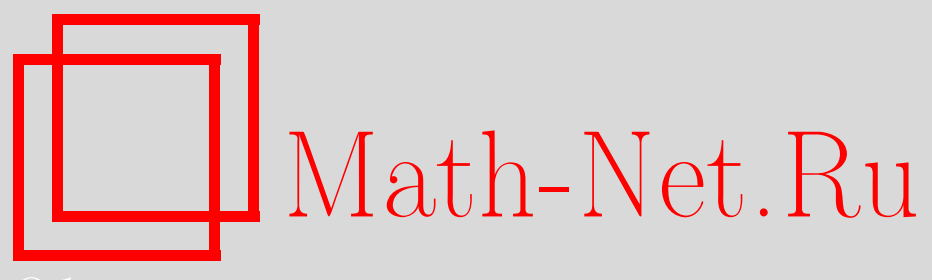

С. В. Конягин, А. А. Кулешов, О непрерывности конечных сумм ридж-функций, Матем. заметки, 2015, том 98, выпуск 2, 308-309

DOI: https://doi.org/10.4213/mzm10787

Использование Общероссийского математического портала Math-Net.Ru подразумевает, что вы прочитали и согласны с пользовательским соглашением http://www . mathnet.ru/rus/agreement

Параметры загрузки:

IP : 52.90 .164 .192

26 апреля 2023 г., 13:45:45

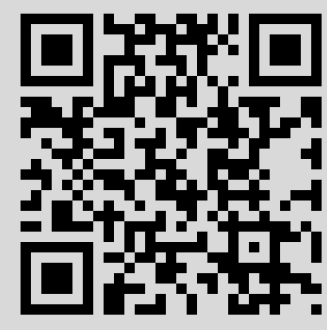




\section{О непрерывности конечных сумм ридж-функций}

\section{С. В. Конягин, А. А. Кулешов}

Пусть $n \geqslant 2, E \subset \mathbb{R}^{n}$ - некоторое множество. Ридж-функиией на $E$ (см. [1]-[4]) будем называть функцию вида $\varphi(\mathbf{a} \cdot \mathbf{x})$, где

$$
\mathbf{x}=\left(x_{1}, \ldots, x_{n}\right) \in E, \quad \mathbf{a}=\left(a_{1}, \ldots, a_{n}\right) \in \mathbb{R}^{n}, \quad \mathbf{a} \cdot \mathbf{x}=\sum_{j=1}^{n} a_{j} x_{j}
$$

и $\varphi$ - функция, определенная на $\Delta(\mathbf{a})=\{\mathbf{a} \cdot \mathbf{x}: \mathbf{x} \in E\}$. На множестве $E$ рассмотрим сумму ридж-функций

$$
f(\mathbf{x})=\sum_{i=1}^{m} \varphi_{i}\left(\mathbf{a}^{i} \cdot \mathbf{x}\right)
$$

Всюду мы будем предполагать, что векторы $\mathbf{a}^{i}$ попарно неколлинеарны и, значит, ненулевые. Обозначим $\Delta_{i}=\Delta\left(\mathbf{a}^{i}\right)$. Нас интересует, вытекает ли из непрерывности $f$ непрерывность функций $\varphi_{i}$. Следующее утверждение основано на результатах [4].

Теорема 1. Пусть множество Е непусто и открыто. Тогда следующие условия равносильны:

1) векторы $\mathbf{a}^{i}$ линейно независимь;

2) из непрерывности на Е функции $f$ вида (1) следует непрерывность функций $\varphi_{i}$ на множествах $\Delta_{i}$.

ДоказАтельство. Пусть выполнено 1). Докажем, что, например, функция $\varphi_{1}$ непрерывна в произвольной точке $t_{0} \in \Delta_{1}$. Зафиксируем точку $\mathbf{x}^{0} \in E$ такую, что $\mathbf{a}^{1} \cdot \mathbf{x}^{0}=t_{0}$. $\mathrm{B}$ силу линейной независимости векторов $\mathbf{a}^{i}$ найдется такой вектор $\mathbf{h} \in \mathbb{R}^{n}$, что $\mathbf{a}^{1} \cdot \mathbf{h}=1$ и $\mathbf{a}^{i} \cdot \mathbf{h}=0$ при $i=2, \ldots, m$. Для $i=2, \ldots, m$ обозначим $\varphi_{i}\left(\mathbf{a}^{i} \cdot \mathbf{x}^{0}\right)$ через $\varphi_{i}^{0}$. Тогда $x^{0}+t \mathbf{h} \in$ $E$ и $\varphi_{i}\left(\mathbf{a}^{i} \cdot\left(\mathbf{x}^{0}+t \mathbf{h}\right)\right)=\varphi_{i}^{0}$ для всех $t$ из некоторой окрестности нуля. Следовательно,

$$
\varphi_{1}\left(t_{0}+t\right)=f\left(x^{0}+t \mathbf{h}\right)-\sum_{i=2}^{m} \varphi_{i}^{0}
$$

откуда вытекает непрерывность $\varphi_{1}$ в $t_{0}$.

Докажем, что из невыполнения 1) следует невыполнение 2). Для этого мы построим определенные на $\mathbb{R}$ функции $\varphi_{i}$, среди которых будут всюду разрывные, так, что (1) выполнено на всем $\mathbb{R}$ для $f \equiv 0$. В силу линейной зависимости векторов найдутся числа $\alpha_{i}$, не все равные нулю, такие, что $\sum_{i=1}^{m} \alpha_{i} \mathbf{a}^{i}=0$. Пусть $\varphi: \mathbb{R} \rightarrow \mathbb{R}$ - произвольная всюду разрывная аддитивная функция (теорема 5.4 , [5]), т.е. $\varphi$ удовлетворяет функциональному уравнению Коши:

$$
\varphi(x+y)=\varphi(x)+\varphi(y) \quad \text { для всех } \quad x, y \in \mathbb{R} .
$$

Тогда функции $\varphi_{i}(t)=\varphi\left(\alpha_{i} t\right)$ обладают требуемым свойством.

Замкнутое выпуклое множество $E \subset \mathbb{R}^{n}$, для которого $\operatorname{int}(E) \neq \varnothing$, называется выпуклым телом. Гиперплоскость $\Gamma=\left\{\mathbf{x} \in \mathbb{R}^{n}: \mathbf{c} \cdot \mathbf{x}=\gamma\right\}$ будем называть опорной к множеству $E$ в точке $\mathbf{y} \in E$, если для всех $\mathbf{x} \in E$ выполнено $\mathbf{c} \cdot \mathbf{x} \geqslant \mathbf{c} \cdot \mathbf{y}=\gamma[6 ; \mathbf{c .} 192]$; тогда $\mathbf{c}$ и $-\mathbf{c}-$ опорные функиионалы множества $E$ в точке у. Границу $\partial(E)$ выпуклого тела $E$ назовем гладкой, если через любую точку $\mathbf{y} \in \partial(E)$ проходит единственная опорная гиперплоскость.

Исследование выполнено за счет гранта Российского научного фонда (проект № 14-50-00005).

DOI: $10.4213 / \mathrm{mzm} 10787$ 
Теорема 2. Пусть $E$ - выпуклое тело в $\mathbb{R}^{n}, E \neq \mathbb{R}^{n}, u m \geqslant 2$. Тогда следующие условия равносильны:

1) граница Е гладкая;

2) для любых векторов $\mathbf{a}^{1}, \ldots, \mathbf{a}^{m}$ и любых функиий $\varphi_{i}$, непрерывных на $\operatorname{int}\left(\Delta_{i}\right), i=$ $1, \ldots, m$, из непрерывности на $E$ функиии $f$ вида (1) следует непрерывность функций $\varphi_{i}$ на множествах $\Delta_{i}$;

3) для любых векторов $\mathbf{a}^{1}, \ldots, \mathbf{a}^{m}$ и любых функиий $\varphi_{i}$, непрерывных на $\operatorname{int}\left(\Delta_{i}\right), i=$ $1, \ldots, m$, из непрерывности на $E$ функции $f$ вида (1) следует локальная ограниченность бункиий $\varphi_{i}$ на множествах $\Delta_{i}$.

ДокАзАтЕльство. Пусть выполнено 1). Докажем, что, например, функция $\varphi_{1}$ непрерывна в граничной точке $t_{0} \in \Delta_{1}$. Зафиксируем точку $\mathbf{x}^{0} \in E$ такую, что $\mathbf{a}^{1} \cdot \mathbf{x}^{0}=t_{0}$. Тогда $\mathbf{x}^{0}$ является граничной точкой множества $E$, а функционал $\mathbf{a}^{1}$ является опорным в этой точке. В силу условия гладкости функционалы $\mathbf{a}^{i}, i \geqslant 2$, не будут опорными в точке $\mathbf{x}^{0}$, т.е. $\mathbf{a}^{i} \cdot \mathbf{x}^{0}$ является внутренней точкой $\Delta_{i}$ и, значит, точкой непрерывности $\varphi_{i}$. Поэтому функция

$$
\varphi_{1}\left(\mathbf{a}^{1} \cdot \mathbf{x}\right)=f(\mathbf{x})-\sum_{i=2}^{m} \varphi_{i}\left(\mathbf{a}^{i} \cdot \mathbf{x}\right)
$$

будет непрерывной в $\mathbf{x}^{0}$, из чего следует требуемое утверждение.

Пусть 1) не выполнено, т.е. имеется точка негладкости $\mathbf{x}^{0}$ на границе $E$. Тогда найдутся неколлинеарные векторы $\mathbf{h}^{1}$ и $\mathbf{h}^{2}$ такие, что

$$
t_{i}=\mathbf{h}^{i} \cdot \mathbf{x}^{0}=\min \left\{\mathbf{h}^{i} \cdot \mathbf{x}: \mathbf{x} \in E\right\}, \quad i=1,2 .
$$

Без ограничения общности можно считать, что $t_{1}=t_{2}=0$. Положим $\mathbf{a}^{1}=\mathbf{h}^{1}+\mathbf{h}^{2}, \mathbf{a}^{2}=$ $\mathbf{h}^{1}+2 \mathbf{h}^{2}$. Пусть $\psi: \mathbb{R} \rightarrow \mathbb{R}$ - произвольная дифференцируемая функция, удовлетворяющая условиям $\lim _{u \rightarrow-\infty} \psi(u)=\infty$ и $\lim _{u \rightarrow-\infty} \psi^{\prime}(u)=0$. Для $i=1,2$ положим $\varphi_{i}(t)=(-1)^{i} \psi(\log t)$ при $t>0$ и $\varphi_{i}(0)=0$, а функция $f$ при $m=2$ определяется равенством (1). Легко видеть, что $f$ будет непрерывной на $E$, а $\left|\varphi_{i}(t)\right| \rightarrow \infty$ при $t \rightarrow 0, i=1,2$. Если $m>2$, то можно дополнительно положить $\mathbf{a}^{i}=\mathbf{h}^{1}+i \mathbf{h}^{2}$ и $\varphi_{i} \equiv 0$ при $i>2$. Теорема доказана.

Теорема 3. Пусть $E$ - выпуклое тело в $\mathbb{R}^{n}$, функиия $f$ вида (1) непрерывна на $E$, функиии $\varphi_{i}$ непрерывны на $\operatorname{int}\left(\Delta_{i}\right), i=1, \ldots, m$, и пусть для некоторого $i$ точка $t_{0} \in \Delta_{i}$ является граничной. Тогда

$$
\varphi_{i}(t)=o\left(-\log \left|t-t_{0}\right|\right) \quad \text { nрu } \quad t \rightarrow t_{0}, \quad t \in \Delta_{i} .
$$

Доказательство теоремы 2 показывает точность логарифмической оценки в теореме 3 .

\section{СПИСОК ЦИТИРОВАННОЙ ЛИТЕРАТУРЫ}

[1] Xingping Sun, E. W. Cheney, Aequationes Math., 44:2-3 (1992), 226-235. [2] D. Braess, A. Pinkus, J. Approx. Theory, 73:2 (1993), 218-236. [3] V. E. Maiorov, J. Approx. Theory, 99:1 (1999), 68-94. [4] A. Pinkus, Indag. Math. (N.S.), 24:4 (2013), 725-738. [5] H. Herrlich, Axiom of Choice, Lecture Notes in Math., 1876, Springer-Verlag, Berlin, 2006. [6] Ф. П. Васильев, Методы оптимизации, Факториал Пресс, М., 2002.

\section{С. В. Конягин}

Поступило

Математический институт им. В. А. Стеклова

16.03.2015

Российской академии наук

E-mail: konyagin23@gmail.com

Исправленный вариант

22.03 .2015

\section{А. А. Кулешов}

Математический институт им. В. А. Стеклова

Российской академии наук

E-mail: kuleshov.a.a@yandex.ru 